\title{
STATISTICAL FIELD THEORY FOR A MULTICOMPONENT FLUID: THE COLLECTIVE VARIABLES APPROACH*
}

\author{
O. Patsahan ${ }^{1}$, I. Mryglod ${ }^{1}$, J.-M. Caillol ${ }^{2}$ \\ ${ }^{1}$ Institute for Condensed Matter Physics, National Academy of Sciences of Ukraine, \\ 1 Svientsitskii St., Lviv, UA-79011, Ukraine \\ ${ }^{2}$ Laboratoire de Physique Théorique CNRS UMR 8627, \\ Bât. 210 Université de Paris-Sud 91405 Orsay Cedex, France
}

(Received January 15, 2007)

\begin{abstract}
Using the collective variables $(\mathrm{CV})$ method the basic relations of statistical field theory of a multicomponent non-homogeneous fluids are reconsidered. The corresponding CV action depends on two sets of scalar fields: the fields $\rho_{\alpha}$ connected to the local density fluctuations of the $\alpha$ th species of particles and those $\omega_{\alpha}$ conjugated to $\rho_{\alpha}$. Explicit expressions for the CV field correlations and their relation to the density correlation functions are found. The perturbation theory is formulated and a mean field level (MF) of this theory is considered in detail.

Key words: theory of liquids, mixtures, statistical field theory, functional methods, collective variables.
\end{abstract}

PACS number(s): 05.70.Fh, 02.70.Rr

\section{INTRODUCTION}

In recent years much attention has been focused on an issue of the phase transitions in multicomponent fluid mixtures, especially in ionic fluids. In spite of significant progress in this field, such systems are far from being completely understood. The investigation of complex models is of great importance in understanding the nature of critical and phase behavior of real ionic fluids which demonstrate both the charge and size asymmetry. The powerful tools for the study of multicomponent continuous systems are those based on the functional methods. In many cases the partition function of multicomponent models (see, e.g. [1]) can be re-expressed as a functional integral after performing the HubbardStratonovich transformation [2,3], a simple device proposed in the 1950s. Nearly at the same time another method, the method of collective variables $(\mathrm{CVs})$, that allows in an explicit way to construct a functional representation for many-particle interacting systems was developed $[4,5]$. The method proposed initially in the $1950 \mathrm{~s}$ [4-6] for the description of the classical charged many particle systems and developed later for the needs of the phase transition theory [7-10] was in fact one of the first successful attempts to address the problems of statistical physics using the functional integral representation. Recently, the rigorous scalar field KSSHE (Kac-SiegertStratonovich-Hubbard-Edwards) theory [11, 12], which uses the Stratonovich-Hubbard transformation, was developed to describe the phase equilibria in simple and ionic fluids. As was shown $[13,14]$, both groups of theories are in close relation.

In [13] the CV representation of simple (onecomponent) fluids was reexamined from the point of view of statistical field theory. Our goal here is to derive the exact functional representation for the grand canonical partition function of a non-homogeneous multicomponent fluid. We reformulate the method of $\mathrm{CV}$ in real space and derive the CV action that depends on two sets of scalar fields: the fields $\left\{\rho_{\alpha}\right\}$ connected to the densities of the $\alpha$ th species and the fields $\left\{\omega_{\alpha}\right\}$ conjugate to $\left\{\rho_{\alpha}\right\}$. We study the correlations between these fields as well as their relations to the density correlations of the fluid.

The CV method is based on: (i) the concept of collective coordinates being appropriate for the physics of the system considered (see, for instance, [15]) and (ii) the integral identity allowing to derive an exact functional representation for the configurational Boltzmann factor. Being applied to the continuous system the $\mathrm{CV}$ method uses the idea of the reference system (RS), one of the basic ideas of the liquid state theory [16]. The idea consists in the splitting of an interparticle interaction potential into two parts: the potential of a short-range repulsion which describes the mutual impenetrability of the particles and the potential describing mainly the behaviour at moderate and large distances. The equilibrium properties of the system interacting via a short-range repulsion are assumed to be known. Therefore, this system can be regarded as the "reference" system. The remainder of the interaction is described in the phase space of CVs (collective coordinates). The fluid of hard spheres is most frequently used as the RS in the liquid state theory since its thermodynamic and structural properties are well known. In this paper we derive the functional representation for the grand canonical partition function of a multicomponent fluid which includes both short-range and long-range interactions. 
The paper is organized as follows. In Section II we obtain the exact expression for the functional of the grand partition function of a multicomponent nonhomogeneous mixture. Section III is devoted to the study of the correlations of $\mathrm{CVs}$ fields and their relation to the density correlation functions of a multicomponent fluid. In Section IV we formulate the perturbation theory. The MF level of the theory is considered in detail.

\section{THE FUNCTIONAL REPRESENTATION OF THE GRAND PARTITION FUNCTION}

\section{A. The model}

Let us consider a classical $m$-component system consisting of $N$ particles among which there exist $N_{1}$ particles of species $1, N_{2}$ particles of species $2, \ldots$ and $N_{m}$ particles of species $m$. The potential energy of the system is assumed to be of the form

$\mathcal{U}_{N_{1} \ldots N_{m}}=\frac{1}{2} \sum_{\alpha, \beta}^{m} \sum_{i \neq j}^{N} U_{\alpha \beta}\left(\mathbf{r}_{i}^{\alpha}, \mathbf{r}_{j}^{\beta}\right)+\sum_{\alpha=1}^{m} \sum_{i=1}^{N} \psi_{\alpha}\left(\mathbf{r}_{i}^{\alpha}\right)$,

where $U_{\alpha \beta}\left(\mathbf{r}_{i}^{\alpha}, \mathbf{r}_{j}^{\beta}\right)$ denotes the interaction potential of two particles and the second term is the potential energy due to external forces.

We present the pair interaction potential $U_{\alpha \beta}\left(\mathbf{r}_{i}^{\alpha}, \mathbf{r}_{j}^{\beta}\right)$ as

$$
U_{\alpha \beta}\left(\mathbf{r}_{i}^{\alpha}, \mathbf{r}_{j}^{\beta}\right)=v_{\alpha \beta}^{0}\left(\mathbf{r}_{i}^{\alpha}, \mathbf{r}_{j}^{\beta}\right)+w_{\alpha \beta}\left(\mathbf{r}_{i}^{\alpha}, \mathbf{r}_{j}^{\beta}\right)
$$

where $v_{\alpha \beta}^{0}\left(\mathbf{r}_{i}^{\alpha}, \mathbf{r}_{j}^{\beta}\right)$ is a potential of a short-range repulsion that can be chosen as an interaction between two hard spheres of respective diameters $\sigma_{\alpha}$ and $\sigma_{\beta}$, respectively we call the $m$-component system with the interaction $v_{\alpha \beta}^{0}\left(\mathbf{r}_{i}^{\alpha}, \mathbf{r}_{j}^{\beta}\right)$ a reference system (RS). The thermodynamic and structural properties of the RS are assumed to be known. $w_{\alpha \beta}\left(\mathbf{r}_{i}^{\alpha}, \mathbf{r}_{j}^{\beta}\right)$ is some potential which can describe both repulsion (e.g. soft repulsion) and attractive interactions. In general, $w_{\alpha \beta}\left(\mathbf{r}_{i}^{\alpha}, \mathbf{r}_{j}^{\beta}\right)$ can be presented in the form

$$
w_{\alpha \beta}\left(\mathbf{r}_{i}^{\alpha}, \mathbf{r}_{j}^{\beta}\right)=w_{\alpha \beta}^{\mathrm{R}}\left(\mathbf{r}_{i}^{\alpha}, \mathbf{r}_{j}^{\beta}\right)+w_{\alpha \beta}^{\mathrm{A}}\left(\mathbf{r}_{i}^{\alpha}, \mathbf{r}_{j}^{\beta}\right),
$$

where $w_{\alpha \beta}^{\mathrm{R}}\left(\mathbf{r}_{i}^{\alpha}, \mathbf{r}_{j}^{\beta}\right)$ and $w_{\alpha \beta}^{\mathrm{A}}\left(\mathbf{r}_{i}^{\alpha}, \mathbf{r}_{j}^{\beta}\right)$ are repulsive and attractive parts of the interaction potential $w_{\alpha \beta}\left(\mathbf{r}_{i}^{\alpha}, \mathbf{r}_{j}^{\beta}\right)$. Since $w_{\alpha \beta}\left(\mathbf{r}_{i}^{\alpha}, \mathbf{r}_{j}^{\beta}\right)$ are arbitrary in the core, i. e. for $r \leq$ $\sigma_{\alpha \beta} \equiv\left(\sigma_{\alpha}+\sigma_{\beta}\right) / 2$, we assume that the $w_{\alpha \beta}\left(\mathbf{r}_{i}^{\alpha}, \mathbf{r}_{j}^{\beta}\right)$ have been regularized in such a way that their Fourier transforms $\widetilde{w}_{\alpha \beta}(k)$ are well-behaved functions of $k_{i}$ and that $w_{\alpha \beta}(0)$ are finite quantities. We denote by $\Omega$ the domain of volume $V$ occupied by particles.

We present the potential energy $\mathcal{U}_{N_{1} \ldots N_{m}}$ as follows

$$
\begin{aligned}
\mathcal{U}_{N_{1} \ldots N_{m}} & =\mathcal{V}_{N_{1} \ldots N_{m}}^{\mathrm{RS}}+\frac{1}{2}\left\langle\widehat{\rho}_{\alpha}\left|w_{\alpha \beta}\right| \widehat{\rho}_{\beta}\right\rangle \\
& +\left\langle\psi_{\alpha} \mid \widehat{\rho}_{\alpha}\right\rangle-N_{\alpha} \nu_{\alpha}^{S}
\end{aligned}
$$

where

$$
\widehat{\rho}_{\alpha}(\mathbf{r})=\sum_{i=1}^{N_{\alpha}} \delta\left(\mathbf{r}-\mathbf{r}_{i}^{\alpha}\right)
$$

is the microscopic density of the $\alpha$ th species in a given configuration and $\psi_{\alpha}(\mathbf{r})$ is some external one-body potential acting on particles of species $\alpha$. The following notations are introduced in (3): $\mathcal{V}_{N_{1} \ldots N_{m}}^{\mathrm{RS}}$ is the contribution from an $m$-component $\mathrm{RS}, \nu_{\alpha}^{\mathrm{S}}$ is the self-energy of the $\alpha$ th species

$$
\nu_{\alpha}^{S}=\frac{1}{2} w_{\alpha \alpha}(0) .
$$

In (3) we have also introduced Dirac's brackets notations

$$
\left\langle\widehat{\rho}_{\alpha}\left|w_{\alpha \beta}\right| \widehat{\rho}_{\beta}\right\rangle=\int_{\Omega} \mathrm{d} \mathbf{r}_{1}^{\alpha} \mathrm{d} \mathbf{r}_{2}^{\beta} \widehat{\rho}_{\alpha}\left(\mathbf{r}_{1}^{\alpha}\right) w_{\alpha \beta}\left(\mathbf{r}_{1}^{\alpha}, \mathbf{r}_{2}^{\beta}\right) \widehat{\rho}_{\beta}\left(\mathbf{r}_{2}^{\beta}\right),
$$

$$
\left\langle\psi_{\alpha} \mid \widehat{\rho}_{\alpha}\right\rangle=\int_{\Omega} \mathrm{d} \mathbf{r}_{1}^{\alpha} \psi_{\alpha}\left(\mathbf{r}_{1}^{\alpha}\right) \widehat{\rho}_{\alpha}\left(\mathbf{r}_{1}^{\alpha}\right)
$$

In the above formulas summation over repeated indices is meant.

The system under consideration is at equilibrium in the grand canonical (GC) ensemble, $\beta=1 / k_{\mathrm{B}} T$ is the inverse temperature ( $k_{\mathrm{B}}$ Boltzmann constant), $\mu_{\alpha}$ is the chemical potential of the $\alpha$ th species. Then, the GC partition function can be written as

$$
\begin{aligned}
\Xi\left[\left\{\nu_{\alpha}\right\}\right] & =\sum_{N_{1} \geq 0} \frac{1}{N_{1} !} \sum_{N_{2} \geq 0} \frac{1}{N_{2} !} \cdots \sum_{N_{m} \geq 0} \frac{1}{N_{m} !} \int(\mathrm{d} \Gamma) \\
& \times \exp \left[-\beta \mathcal{V}_{N_{1} \ldots N_{m}}^{\mathrm{RS}}-\frac{\beta}{2}\left\langle\widehat{\rho}_{\alpha}\left|w_{\alpha \beta}\right| \widehat{\rho}_{\beta}\right\rangle+\left\langle\bar{\nu}_{\alpha} \mid \widehat{\rho}_{\alpha}\right\rangle\right]
\end{aligned}
$$

where $\bar{\nu}_{\alpha}(\mathbf{r})=\nu_{\alpha}+\nu_{\alpha}^{S}-\beta \psi_{\alpha}(\mathbf{r})$ is the local chemical potential of the $\alpha$ th species and $\nu_{\alpha}=\beta \mu_{\alpha}-3 \ln \Lambda_{\alpha}, \Lambda_{\alpha}^{-1}=$ $\left(2 \pi m_{\alpha} \beta^{-1} / h^{2}\right)^{1 / 2}$ is the inverse de Broglie thermal wavelength. $(\mathrm{d} \Gamma)=\prod_{\alpha} \mathrm{d} \Gamma_{\mathrm{N}_{\alpha}}, \mathrm{d} \Gamma_{N_{\alpha}}=d \mathbf{r}_{1}^{\alpha} \mathrm{d} \mathbf{r}_{2}^{\alpha} \ldots \mathrm{d} \mathbf{r}_{N_{\alpha}}^{\alpha}$ is the element of the configurational space of $N$ particles.

For a given volume $V, \Xi\left[\nu_{\alpha}\right]$ is a function of the temperature $T$ and a log-convex functional of the local chemical potentials $\nu_{\alpha}\left(\mathbf{r}^{\alpha}\right)$.

\section{B. The collective variables representation}

We introduce the collective variable $\rho_{\alpha}(\mathbf{r})$ which describes the field of the number particle density of the $\alpha$ th species. To this end we use the identity

$$
\begin{aligned}
\exp \left(\frac{1}{2}\langle\widehat{\rho}|w| \widehat{\rho}\rangle\right) & =\int \mathcal{D} \rho \delta_{\mathcal{F}}[\rho-\widehat{\rho}] \\
& \times \exp \left(\frac{1}{2}\langle\rho|w| \rho\rangle\right)
\end{aligned}
$$

In $(7)$ the functional "delta function" $\delta_{\mathcal{F}}[\rho]$ is defined as [17] 


$$
\delta_{\mathcal{F}}[\rho] \equiv \int \mathcal{D} \omega \exp (i\langle\omega \mid \rho\rangle)
$$

Using (8) we can present the Boltzmann factor which does not include the RS interaction in the form

$$
\begin{aligned}
& \exp \left(\frac{1}{2}\left\langle\widehat{\rho}_{\alpha}\left|w_{\alpha \beta}\right| \widehat{\rho}_{\beta}\right\rangle\right)=\int \mathcal{D} \rho \mathcal{D} \omega \\
& \times \exp \left(\frac{1}{2}\left\langle\rho_{\alpha}\left|w_{\alpha \beta}\right| \rho_{\beta}\right\rangle+\mathrm{i}\left\langle\omega_{\alpha} \mid\left\{\rho_{\alpha}-\widehat{\rho}_{\alpha}\right\}\right\rangle\right) .
\end{aligned}
$$

Inserting equation (9) in definition (7) of the GC partition function $\Xi\left[\nu_{\alpha}\right]$ one obtains

$$
\begin{aligned}
\Xi\left[\left\{\nu_{\alpha}\right\}\right] & =\int \mathcal{D} \rho \exp \left(-\frac{\beta}{2}\left\langle\rho_{\alpha}\left|w_{\alpha \beta}\right| \rho_{\beta}\right\rangle\right) \\
& \times \mathcal{J}\left[\left\{\rho_{\alpha}, \bar{\nu}_{\alpha}\right\}\right]
\end{aligned}
$$

where the Jacobian

$$
\begin{aligned}
\mathcal{J}\left[\left\{\rho_{\alpha}, \bar{\nu}_{\alpha}\right\}\right] & =\int \mathcal{D} \omega \exp \left(\mathrm{i}\left\langle\omega_{\alpha} \mid \rho_{\alpha}\right\rangle\right) \\
& \times \Xi_{R S}\left[\left\{\bar{\nu}_{\alpha}-\mathrm{i} \omega_{\alpha}\right\}\right]
\end{aligned}
$$

allows one for the passage from the microscopic variables $\mathbf{r}^{\alpha}$ (the Cartesian coordinates of particles) to the collective variables $\rho_{\alpha}(\mathbf{r})$ (fields of the number density of particles). In (11) $\Xi_{\mathrm{RS}}\left[\left\{\bar{\nu}_{\alpha}-\mathrm{i} \omega_{\alpha}\right\}\right]=\Xi_{\mathrm{RS}}\left[Z^{*}\right]$ is the GC partition function of an $m$-component RS

$$
\begin{aligned}
\Xi_{\mathrm{RS}}\left[Z^{*}\right] & =\sum_{N_{1} \geq 0} \frac{1}{N_{1} !} \sum_{N_{2} \geq 0} \frac{1}{N_{2} !} \ldots \sum_{N_{m} \geq 0} \frac{1}{N_{m} !} \int(\mathrm{d} \Gamma) \\
& \times \exp \left(-\beta \mathcal{V}_{N_{1} \ldots N_{m}}^{\mathrm{RS}}\right) \prod_{i} Z^{*}\left(\mathbf{r}_{i}\right)
\end{aligned}
$$

where $Z^{*}(\mathbf{r})=\prod_{\alpha} Z_{\alpha}^{*}(\mathbf{r})=\exp \left(\nu_{\alpha}^{*}(\mathbf{r})\right) . Z_{\alpha}^{*}$ is the activity of the species $\alpha$ associated with the dimensionless local chemical potential $\nu_{\alpha}^{*}(\mathbf{r})=\bar{\nu}_{\alpha}(r)-\mathrm{i} \omega_{\alpha}(\mathbf{r})$. It should be noted that $\mathcal{J}\left[\left\{\rho_{\alpha}, \bar{\nu}_{\alpha}\right\}\right]$ does not depend on the pair interaction $w_{\alpha \beta}\left(\mathbf{r}_{i}, \mathbf{r}_{j}\right)$ but only on the GC partition function of the $\mathrm{RS} \Xi_{\mathrm{RS}}\left[Z^{*}\right]$ which is supposed to be known.

Equation (10) can also easily be recast in the form of a standard statistical field theory, i. e. as

$$
\Xi\left[\left\{\nu_{\alpha}\right\}\right]=\int \mathcal{D} \rho \mathcal{D} \omega \exp \left(-\mathcal{H}\left[\left\{\nu_{\alpha}, \rho_{\alpha}, \omega_{\alpha}\right\}\right]\right),
$$

where the action $\mathcal{H}\left[\left\{\nu_{\alpha}, \rho_{\alpha}, \omega_{\alpha}\right\}\right]$ of the CV field theory reads as

$$
\begin{aligned}
\mathcal{H}\left[\left\{\nu_{\alpha}, \rho_{\alpha}, \omega_{\alpha}\right\}\right] & =\frac{\beta}{2}\left\langle\rho_{\alpha}\left|w_{\alpha \beta}\right| \rho_{\beta}\right\rangle-\mathrm{i}\left\langle\omega_{\alpha} \mid \rho_{\alpha}\right\rangle \\
& -\ln \Xi_{\mathrm{RS}}\left[\left\{\bar{\nu}_{\alpha}-\mathrm{i} \omega_{\alpha}\right\}\right] .
\end{aligned}
$$

Functional integrals which enter the above-mentioned formulas can be given a precise meaning in the case where the domain $\Omega$ is a cube of side $L\left(V=L^{3}\right)$ with periodic boundary conditions. This means that we restrict ourselves to the fields $\rho_{\alpha}(\mathbf{r})$ and $\omega_{\alpha}(\mathbf{r})$ which can be written as Fourier series

$$
\rho_{\alpha}(\mathbf{r})=\frac{1}{L^{3}} \sum_{\mathbf{k} \in \boldsymbol{\Lambda}} \rho_{\mathbf{k}, \alpha} e^{\mathrm{i} \mathbf{k r}}
$$

and

$$
\omega_{\alpha}(\mathbf{r})=\frac{1}{L^{3}} \sum_{\mathbf{k} \in \boldsymbol{\Lambda}} \omega_{\mathbf{k}, \alpha} e^{i \mathbf{k r}}
$$

where $\Lambda=(2 \pi / L) \mathbb{Z}^{3}$ is the reciprocal cubic lattice. The reality of $\rho_{\alpha}$ (and $\omega_{\alpha}$ ) implies that, for $\mathbf{k} \neq 0$ $\rho_{-\mathbf{k}, \alpha}=\rho_{\mathbf{k}, \alpha}^{\star}\left(\omega_{-\mathbf{k}, \alpha}=\omega_{\mathbf{k}, \alpha}^{\star}\right)$, where the star means complex conjugation. Then the normalized functional measure $\mathcal{D} \rho($ and $\mathcal{D} \omega)$ is defined as [18]

$$
\begin{aligned}
\mathcal{D} \rho & \equiv \prod_{\alpha} \prod_{\mathbf{k} \in \boldsymbol{\Lambda}} \frac{\mathrm{d} \rho_{\mathbf{k}, \alpha}}{\sqrt{2 \pi V}} \\
\mathrm{~d} \rho_{\mathbf{k}, \alpha} \mathrm{d} \rho_{-\mathbf{k}, \alpha} & =2 \mathrm{~d} \Re \rho_{\mathbf{k}, \alpha} \mathrm{d} \Im \rho_{\mathbf{k}, \alpha}, \quad \mathbf{k} \neq \mathbf{0} .
\end{aligned}
$$

Equation (17) can be rewritten as

$$
\mathcal{D} \rho=\prod_{\alpha} \frac{\mathrm{d} \rho_{0, \alpha}}{\sqrt{2 \pi V}} \prod_{\mathbf{q} \in \Lambda^{\star}} \frac{\mathrm{d} \Re \rho_{\mathbf{q}, \alpha} \mathrm{d} \Im \rho_{\mathbf{q}, \alpha}}{\pi V}
$$

where the sum in the r.h.s. runs over only the half $\Lambda^{\star}$ of all the vectors of the reciprocal lattice $\Lambda$. We have for $\mathcal{D} \omega$, respectively

$$
\mathcal{D} \omega=\prod_{\alpha} \frac{\mathrm{d} \omega_{0, \alpha}}{\sqrt{2 \pi V}} \prod_{\mathbf{q} \in \Lambda^{\star}} \frac{\mathrm{d} \Re \omega_{\mathbf{q}, \alpha} \mathrm{d} \Im \omega_{\mathbf{q}, \alpha}}{\pi V} .
$$

Now let us present action (14) for the isotropic interaction potential $w_{\alpha \beta}(r)$ as follows

$$
\begin{aligned}
& \mathcal{H}\left[\left\{\nu_{\alpha}, \rho_{\alpha}, \omega_{\alpha}\right\}\right]=\frac{1}{2} \sum_{\alpha, \beta} \sum_{\mathbf{k}} \widetilde{\Phi}_{\alpha \beta}(k) \rho_{\mathbf{k}, \alpha} \rho_{-\mathbf{k}, \beta} \\
& -\mathrm{i} \sum_{\alpha} \sum_{\mathbf{k}} \omega_{\mathbf{k}, \alpha} \rho_{\mathbf{k}, \alpha}-\ln \Xi_{\mathrm{RS}}\left[\left\{\bar{\nu}_{\alpha}-\mathrm{i} \omega_{\alpha}\right\}\right],
\end{aligned}
$$

Here CV $\rho_{\mathbf{k}, \alpha}$ describes the $k$ th mode of number density fluctuations of the $\alpha$ th species. $\widetilde{\Phi}_{\alpha \beta}(k)=\frac{\beta}{V} \widetilde{w}_{\alpha \beta}(k)$, where $\widetilde{w}_{\alpha \beta}(k)$ is the Fourier transform of the interaction potential $w_{\alpha \beta}(r)$.

In order to obtain another equivalent representation of the action $\mathcal{H}\left[\left\{\nu_{\alpha}, \rho_{\alpha}, \omega_{\alpha}\right\}\right]$ we first distinguish the chemical potential $\nu_{\alpha}^{0}$ of the particle of the species $\alpha$ in the RS. To this end we put

$$
\bar{\nu}_{\alpha}-\mathrm{i} \omega_{\alpha}(\mathbf{r})=\nu_{\alpha}^{0}-\mathrm{i} \omega_{\alpha}{ }^{\prime}(\mathbf{r})
$$

and obtain

$$
\mathrm{i} \omega_{\alpha}(\mathbf{r})=\Delta \nu_{\alpha}+\mathrm{i} \omega_{\alpha}^{\prime}(\mathbf{r})
$$

with $\Delta \nu_{\alpha}=\bar{\nu}_{\alpha}-\nu_{\alpha}^{0}$. As a result, the action (or the Hamiltonian) (21) can be written as 


$$
\mathcal{H}\left[\left\{\nu_{\alpha}, \rho_{\alpha}, \omega_{\alpha}\right\}\right]=-\sum_{\alpha} \Delta \nu_{\alpha} \rho_{\mathbf{k}=\mathbf{0}, \alpha}+\frac{1}{2} \sum_{\alpha, \beta} \sum_{\mathbf{k}} \widetilde{\Phi}_{\alpha \beta}(k) \rho_{\mathbf{k}, \alpha} \rho_{-\mathbf{k}, \beta}-\mathrm{i} \sum_{\alpha} \sum_{\mathbf{k}} \omega_{\mathbf{k}, \alpha}{ }^{\prime} \rho_{\mathbf{k}, \alpha}-\ln \Xi_{\mathrm{RS}}\left[\left\{\nu_{\alpha}^{0}-\mathrm{i} \omega_{\alpha}^{\prime}\right\}\right] .
$$

We have obtained the exact representations of the grand partition function of a multicomponent system (eqs. (13)-(14), (21) and (23)) in terms of CVs $\rho_{\alpha}(\mathbf{r})$, which are number density fields of the $\alpha$ th species particles (or fluctuation modes of the $\alpha$ th species number density) We also stress that $\rho_{\alpha}(\mathbf{r})$ and $\omega_{\alpha}(\mathbf{r})$ are two real scalar fields and that eqs. (13)-(14) (as well as eqs. (21) and (23)) are valid for repulsive, attractive as well as arbitrary pair interactions.

Besides the usual GC average $\langle\mathcal{A}\rangle_{\mathrm{GC}}$ of a dynamic variable we introduce statistical field averages of the type

$$
\left\langle\mathcal{A}\left[\left\{\rho_{\alpha}, \omega_{\alpha}\right\}\right]\right\rangle_{\mathrm{CV}}=\Xi\left[\left\{\nu_{\alpha}\right\}\right]^{-1} \int \mathcal{D} \rho \mathcal{D} \omega \mathcal{A}\left[\left\{\rho_{\alpha}, \omega_{\alpha}\right\}\right] \exp \left(-\mathcal{H}\left[\left\{\nu_{\alpha}, \rho_{\alpha}, \omega_{\alpha}\right\}\right]\right),
$$

where $\mathcal{A}\left[\left\{\rho_{\alpha}, \omega_{\alpha}\right\}\right]$ is a functional of the two CV fields $\rho_{\alpha}$ and $\omega_{\alpha}$.

\section{CORRELATION FUNCTIONS}

\section{A. General relations}

Let us write some important relations. First, according to [20,21] we introduce the ordinary and truncated (or connected) density correlation functions

$$
\begin{aligned}
& G_{\alpha_{1} \ldots \alpha_{n}}^{(n)}\left[\left\{\nu_{\alpha}\right\}\right](1, \ldots, n)=\left\langle\prod_{1=1}^{n} \widehat{\rho}_{\alpha_{i}}(i)\right\rangle_{\mathrm{GC}}=\frac{1}{\Xi\left[\nu_{\alpha}\right]} \frac{\delta^{n} \Xi\left[\left\{\nu_{\alpha}\right\}\right]}{\delta \nu_{\alpha_{1}}(1) \ldots \delta \nu_{\alpha_{n}}(n)}, \\
& G_{\alpha_{1} \ldots \alpha_{n}}^{(n), T}\left[\left\{\nu_{\alpha}\right\}\right](1, \ldots, n)=\frac{\delta^{n} \log \Xi\left[\left\{\nu_{\alpha}\right\}\right]}{\delta \nu_{\alpha_{1}}(1) \ldots \delta \nu_{\alpha_{n}}(n)} .
\end{aligned}
$$

Our notation emphasizes the fact that the correlation functions (connected and not connected) are functionals of the local chemical potential $\nu_{\alpha}(\mathbf{r})$ and functions of the coordinates $(1,2, \ldots, n) \equiv\left(\mathbf{r}_{1}, \mathbf{r}_{2}, \ldots, \mathbf{r}_{n}\right)$. For the sake of simplicity, we omit below the notations which indicate the functional dependence of the correlation functions of $\nu_{\alpha}(\mathbf{r})$. In standard textbooks of liquid theory [16] the $n$-particle correlation functions are more frequently defined as functional derivatives of $\Xi$ or $\log \Xi$ with respect to the activities $Z_{\alpha}=\exp \left(\nu_{\alpha}\right)$ rather than with respect to the local chemical potentials

$$
\begin{aligned}
& \frac{\rho_{\alpha_{1} \ldots \alpha_{n}}(1,2, \ldots, n)}{Z_{\alpha_{1}}^{*}(1) Z_{\alpha_{2}}^{*}(2) \ldots Z_{\alpha_{n}}^{*}(n)}=\frac{1}{\Xi} \frac{\delta^{n} \Xi}{\delta Z_{\alpha_{1}}^{*}(1) \delta Z_{\alpha_{2}}^{*}(2) \ldots \delta Z_{\alpha_{n}}^{*}(n)}, \\
& \frac{\rho_{\alpha_{1} \ldots \alpha_{n}}^{T}(1,2, \ldots, n)}{Z_{\alpha_{1}}^{*}(1) Z_{\alpha_{2}}^{*}(2) \ldots Z_{\alpha_{n}}^{*}(n)}=\frac{1}{\Xi} \frac{\delta^{n} \ln \Xi}{\delta Z_{\alpha_{1}}^{*}(1) \delta Z_{\alpha_{2}}^{*}(2) \ldots \delta Z_{\alpha_{n}}^{*}(n)} .
\end{aligned}
$$

We also define the partial distribution function $g_{\alpha_{1} \ldots \alpha_{n}}(1,2, \ldots, n)$ and the partial correlation functions $h_{\alpha_{1} \ldots \alpha_{n}}(1,2, \ldots, n)[20]$

$$
g_{\alpha_{1} \ldots \alpha_{n}}(1,2, \ldots, n)=\frac{\rho_{\alpha_{1} \ldots \alpha_{n}}(1,2, \ldots, n)}{\rho_{\alpha_{1}}(1) \rho_{\alpha_{2}}(2) \ldots \rho_{\alpha_{n}}(n)}
$$

and

$$
h_{\alpha_{1} \ldots \alpha_{n}}(1,2, \ldots, n)=\frac{\rho_{\alpha_{1} \ldots \alpha_{n}}^{T}(1,2, \ldots, n)}{\rho_{\alpha_{1}}(1) \rho_{\alpha_{2}}(2) \ldots \rho_{\alpha_{n}}(n)} .
$$

Expressions (25) and (28)-(29) differ by the terms involving products of delta functions. For instance, for $n=2$ and for a homogeneous system one has

$$
\begin{gathered}
G_{\alpha \beta}^{(2)}(1,2)=\rho_{\alpha} \rho_{\beta} g_{\alpha \beta}(1,2)+\rho_{\alpha} \delta_{\alpha \beta} \delta(1,2), \\
G_{\alpha \beta}^{(2), T}(1,2)=\rho_{\alpha} \rho_{\beta} h_{\alpha \beta}(1,2)+\rho_{\alpha} \delta_{\alpha \beta} \delta(1,2),
\end{gathered}
$$

where $\rho_{\alpha}$ is the equilibrium density of the species $\alpha$. Besides, functions $h_{\alpha \beta}(r)$ and $g_{\alpha \beta}(r)$ are connected by the relation $h_{\alpha \beta}(r)=g_{\alpha \beta}(r)-1$ for a homogeneous case. 


\section{B. CV field correlations}

Let us consider the correlations of fields $\rho_{\alpha}$ and $\omega_{\alpha}$. We start with the definitions of the correlation functions

$$
\begin{aligned}
& G_{\rho_{\alpha_{1}} \ldots \rho_{\alpha_{n}}}^{(n)}\left[\left\{\nu_{\alpha}\right\}\right](1, \ldots, n)=\left\langle\prod_{i=1}^{n} \rho_{\alpha_{i}}(i)\right\rangle_{\mathrm{CV}}, \\
& G_{\omega_{\alpha_{1} \ldots \omega_{\alpha_{n}}}}^{(n)}\left[\left\{\nu_{\alpha}\right\}\right](1, \ldots, n)=\left\langle\prod_{i=1}^{n} \omega_{\alpha_{i}}(i)\right\rangle_{\mathrm{CV}}
\end{aligned}
$$

and their truncated (connected) parts

$$
\begin{aligned}
& G_{\rho_{\alpha_{1} \ldots \rho_{\alpha_{n}}}}^{(n), T}(1, \ldots, n)=G_{\rho_{\alpha_{1} \ldots \rho_{\alpha_{n}}}}^{(n)}(1, \ldots, n) \\
& -\sum \prod_{m<n} G_{\rho_{\alpha_{1}} \ldots \rho_{\alpha_{m}}}^{(m), T}\left(i_{1}, \ldots, i_{m}\right), \\
& G_{\omega_{\alpha_{1} \ldots \omega_{\alpha_{n}}}}^{(n), T}(1, \ldots, n)=G_{\omega_{\alpha_{1} \ldots \omega_{\alpha_{n}}}}^{(n)}(1, \ldots, n) \\
& -\sum \prod_{m<n} G_{\omega_{\alpha_{1} \ldots \omega_{\alpha_{m}}}}^{(m), T}\left(i_{1}, \ldots, i_{m}\right),
\end{aligned}
$$

where the sum of products is carried out over all possible partitions of the set $(1, \ldots, n)$ into subsets of cardinality $m<n$.

a. Correlation functions $G_{\rho_{\alpha_{1}} \ldots \rho_{\alpha_{n}}}^{(n)}$. Now we introduce the modified partition function

$$
\Xi^{1}\left[\left\{\nu_{\alpha}, J_{\alpha}\right\}\right]=\int \mathcal{D} \rho \mathcal{D} \omega \exp \left(-\mathcal{H}_{\mathrm{CV}}\left[\left\{\nu_{\alpha}, \rho_{\alpha}, \omega_{\alpha}\right\}\right]+\left\langle J_{\alpha} \mid \rho_{\alpha}\right\rangle\right)
$$

where $J_{\alpha}$ is a real scalar field and $\Xi^{1}\left[\left\{\nu_{\alpha}, J_{\alpha}\right\}\right]$ is the generator of field correlation functions $G_{\rho_{\alpha_{1}} \ldots \rho_{\alpha_{n}}}^{(n)}[19]$. As a result, we have

$$
\begin{aligned}
& G_{\rho_{\alpha_{1}} \ldots \rho_{\alpha_{n}}}^{(n)}\left[\left\{\nu_{\alpha}\right\}\right](1, \ldots, n)=\left.\frac{1}{\Xi^{1}\left[\left\{\nu_{\alpha}\right\}\right]} \frac{\delta^{n} \Xi^{1}\left[\left\{\nu_{\alpha}, J_{\alpha}\right\}\right]}{\delta J_{\alpha_{1}}(1) \ldots \delta J_{\alpha_{n}}(n)}\right|_{J_{\alpha_{i}}=0} \\
& G_{\rho_{\alpha_{1}} \ldots \rho_{\alpha_{n}}}^{(n), T}\left[\left\{\nu_{\alpha}\right\}\right](1, \ldots, n)=\left.\frac{\delta^{n} \log \Xi^{1}\left[\left\{\nu_{\alpha}, J_{\alpha}\right\}\right]}{\delta J_{\alpha_{1}}(1) \ldots \delta J_{\alpha_{n}}(n)}\right|_{J_{\alpha_{i}}=0}
\end{aligned}
$$

The simplest way to obtain the relations between the $G_{\rho_{\alpha_{1}} \ldots \rho_{\alpha_{n}}}^{(n)}\left[\left\{\nu_{\alpha}\right\}\right](1, \ldots, n)$ and the density correlation functions is to start from definition (25). One has

$$
\begin{aligned}
& G_{\alpha_{1} \ldots \alpha_{n}}^{(n)}(1, \ldots, n)=\frac{1}{\Xi\left[\left\{\nu_{\alpha}\right\}\right]} \frac{\delta^{n} \Xi\left[\left\{\nu_{\alpha}\right\}\right]}{\delta \nu_{\alpha_{1}}(1) \ldots \delta \nu_{\alpha_{n}}(n)}=\frac{1}{\Xi\left[\left\{\nu_{\alpha}\right\}\right]} \int \mathcal{D} \rho \mathcal{D} \omega \exp \left(\frac{1}{2}\left\langle\rho_{\alpha}\left|w_{\alpha \beta}^{*}\right| \rho_{\beta}\right\rangle\right. \\
& \left.+\mathrm{i}\left\langle\omega_{\alpha} \mid \rho_{\alpha}\right\rangle\right) \frac{\delta^{n} \Xi_{\mathrm{RS}}\left[\left\{\bar{\nu}_{\alpha}-\mathrm{i} \omega_{\alpha}\right\}\right]}{\delta \nu_{\alpha_{1}}(1) \ldots \delta \nu_{\alpha_{n}}(n)}=\frac{1}{\Xi\left[\left\{\nu_{\alpha}\right\}\right]} \int \mathcal{D} \rho \mathcal{D} \omega \exp \left(\frac{1}{2}\left\langle\rho_{\alpha}\left|w_{\alpha \beta}^{*}\right| \rho_{\beta}\right\rangle+\mathrm{i}\left\langle\omega_{\alpha} \mid \rho_{\alpha}\right\rangle\right)(\mathrm{i})^{n} \frac{\delta^{n} \Xi_{\mathrm{RS}}\left[\left\{\bar{\nu}_{\alpha}-\mathrm{i} \omega_{\alpha}\right\}\right]}{\delta \omega_{\alpha_{1}}(1) \ldots \delta \omega_{\alpha_{n}}(n)},
\end{aligned}
$$

where we introduce the notation $w_{\alpha \beta}^{*}=-\beta w_{\alpha \beta}$ and use the equality

$$
\frac{\delta^{n} \Xi_{\mathrm{RS}}\left[\left\{\bar{\nu}_{\alpha}-\mathrm{i} \omega_{\alpha}\right\}\right]}{\delta \nu_{\alpha_{1}}(1) \ldots \delta \nu_{\alpha_{n}}(n)}=(\mathrm{i})^{n} \frac{\delta^{n} \Xi_{\mathrm{RS}}\left[\left\{\bar{\nu}_{\alpha}-\mathrm{i} \omega_{\alpha}\right\}\right]}{\delta \omega_{\alpha_{1}}(1) \ldots \delta \omega_{\alpha_{n}}(n)} .
$$

Performing now $n$ integral by parts yields

$$
\begin{aligned}
G_{\alpha_{1} \ldots \alpha_{n}}^{(n)}(1, \ldots, n) & =\frac{(-\mathrm{i})^{n}}{\Xi\left[\left\{\nu_{\alpha}\right\}\right]} \int \mathcal{D} \rho \mathcal{D} \omega \exp \left(\frac{1}{2}\left\langle\rho_{\alpha}\left|w_{\alpha \beta}^{*}\right| \rho_{\beta}\right\rangle+\ln \Xi_{\mathrm{RS}}\left[\left\{\bar{\nu}_{\alpha}-\mathrm{i} \omega_{\alpha}\right\}\right]\right) \frac{\delta^{n} \exp \left(\mathrm{i}\left\langle\omega_{\alpha} \mid \rho_{\alpha}\right\rangle\right)}{\delta \omega_{\alpha_{1}}(1) \ldots \delta \omega_{\alpha_{n}}(n)} \\
& =\left\langle\prod_{i=1}^{n} \rho_{\alpha_{i}}(i)\right\rangle_{\mathrm{CV}}
\end{aligned}
$$

We have just proved the expected result

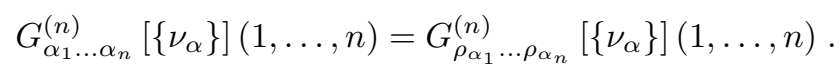

Obviously, the following relation is also valid for the truncated (connected) correlation functions

$$
G_{\alpha_{1} \ldots \alpha_{n}}^{(n), T}\left[\left\{\nu_{\alpha}\right\}\right](1, \ldots, n)=G_{\rho_{\alpha_{1} \ldots \rho_{\alpha_{n}}}}^{(n), T}\left[\left\{\nu_{\alpha}\right\}\right](1, \ldots, n) .
$$


b. Correlation functions $G_{\omega_{\alpha_{1}} \ldots \omega_{\alpha_{n}}}^{(n)}$. Let us define the modified partition function

$$
\Xi^{2}\left[\left\{\nu_{\alpha}, J_{\alpha}\right\}\right]=\int \mathcal{D} \rho \mathcal{D} \omega \exp \left(-\mathcal{H}_{\mathrm{CV}}\left[\left\{\nu_{\alpha}, \rho_{\alpha}, \omega_{\alpha}\right\}\right]+\left\langle J_{\alpha} \mid \omega_{\alpha}\right\rangle\right)
$$

where $J_{\alpha}$ is a real scalar field. $\Xi^{2}\left[\left\{\nu_{\alpha}, J_{\alpha}\right\}\right]$ is the generator of the functions $G_{\omega_{\alpha_{1}} \ldots \omega_{\alpha_{n}}}^{(n)}$ and we thus have

$$
\begin{aligned}
& G_{\omega_{\alpha_{1} \ldots \omega_{\alpha_{n}}}^{(n)}}^{(n)}\left[\left\{\nu_{\alpha}\right\}\right](1, \ldots, n)=\left.\frac{1}{\Xi^{2}\left[\left\{\nu_{\alpha}\right]\right\}} \frac{\delta^{n} \Xi^{2}\left[\left\{\nu_{\alpha}, J_{\alpha}\right\}\right]}{\delta J_{\alpha_{1}}(1) \ldots \delta J_{\alpha_{n}}(n)}\right|_{J_{\alpha_{i}}=0}, \\
& G_{\omega_{\alpha_{1} \ldots \omega_{\alpha_{n}}}^{(n), T}}\left[\left\{\nu_{\alpha}\right\}\right](1, \ldots, n)=\left.\frac{\delta^{n} \log \Xi^{2}\left[\left\{\nu_{\alpha}, J_{\alpha}\right\}\right]}{\delta J_{\alpha_{1}}(1) \ldots \delta J_{\alpha_{n}}(n)}\right|_{J_{\alpha_{i}}=0} .
\end{aligned}
$$

In order to relate the correlation functions $G_{\omega_{\alpha_{1}} \ldots \omega_{\alpha_{n}}}^{(n)}$ and $G_{\alpha_{1} \ldots \alpha_{n}}^{(n)}(1, \ldots, n)$ we perform the change of variables $\rho_{\alpha} \rightarrow \rho_{\alpha}+\mathrm{i} J_{\alpha}$ in Eq. (38). The functional Jacobian of the transformation is of course equal to unity and one obtains the relation

$$
\ln \Xi^{2}\left[\left\{\nu_{\alpha}, J_{\alpha}\right\}\right]=-\frac{1}{2}\left\langle J_{\alpha}\left|w_{\alpha \beta}^{*}\right| J_{\beta}\right\rangle+\ln \Xi^{1}\left[\left\{\nu_{\alpha}, \mathrm{i} w_{\alpha \beta}^{*} \star J_{\beta}\right\}\right],
$$

where the star $\star$ means space convolution and $\Xi^{1}$ is defined in (33). The idea is to perform now $n$ successive functional derivatives of both sides of Eq. (40) with respect to $J_{\alpha}$. Since it follows from expression (34) that

$$
\left.\frac{\delta^{n} \log \Xi^{1}\left[\left\{\nu_{\alpha}, \mathrm{i} w_{\alpha \beta}^{*} \star J_{\beta}\right\}\right]}{\delta J_{\alpha_{1}}(1) \ldots \delta J_{\alpha_{n}}(n)}\right|_{J_{\alpha_{i}}=0}=\mathrm{i}^{n} w_{\alpha_{1} \alpha_{1^{\prime}}}^{*}\left(1,1^{\prime}\right) \ldots w_{\alpha_{n} \alpha_{n^{\prime}}}^{*}\left(n, n^{\prime}\right) G_{\rho_{\alpha_{1^{\prime}}} \ldots \rho_{\alpha_{n^{\prime}}}}^{(n), T}\left[\left\{\nu_{\alpha}\right\}\right]\left(1^{\prime}, \ldots, n^{\prime}\right),
$$

one obtains

$$
\begin{aligned}
& \left\langle\omega_{\alpha_{1}}(1)\right\rangle_{\mathrm{CV}}=\mathrm{i} w_{\alpha_{1} \alpha_{1^{\prime}}}^{*}\left(1,1^{\prime}\right)\left\langle\rho_{\alpha_{1^{\prime}}}\left(1^{\prime}\right)\right\rangle_{\mathrm{CV}}, \\
& G_{\omega_{\alpha_{1}} \omega_{\alpha_{2}}}^{(2), T}\left[\left\{\nu_{\alpha}\right\}\right](1,2)=-w_{\alpha_{1} \alpha_{2}}^{*}(1,2)-w_{\alpha_{1} \alpha_{1^{\prime}}}^{*}\left(1,1^{\prime}\right) w_{\alpha_{2} \alpha_{2^{\prime}}}^{*}\left(2,2^{\prime}\right), \quad G_{\rho_{\alpha_{\alpha^{\prime}}} \rho_{\alpha_{2^{\prime}}}}^{(2), T}\left[\left\{\nu_{\alpha}\right\}\right]\left(1^{\prime}, 2^{\prime}\right), \\
& G_{\omega_{\alpha_{1}} \ldots \omega_{\alpha_{n}}}^{(n), T}\left[\left\{\nu_{\alpha}\right\}\right](1, \ldots, n)=\mathrm{i}^{n} w_{\alpha_{1} \alpha_{1^{\prime}}}^{*}\left(1,1^{\prime}\right) \ldots w_{\alpha_{n} \alpha_{n^{\prime}}}^{*}\left(n, n^{\prime}\right) G_{\rho_{\alpha_{1^{\prime}}} \ldots \rho_{\alpha_{n^{\prime}}}}^{(n), T}\left[\left\{\nu_{\alpha}\right\}\right]\left(1^{\prime}, \ldots, n^{\prime}\right), \quad n \geq 3 .
\end{aligned}
$$

\section{THE PERTURBATION THEORY}

\section{A. Mean-field theory}

Let us consider the functional of the GC partition function (13) with the action given by Eq. (21) for the case of an isotropic interaction potential $w_{\alpha \beta}(r)$. At the MF level one has [19]

$$
\Xi_{\mathrm{MF}}\left[\left\{\nu_{\alpha}\right\}\right]=\exp \left(-\mathcal{H}\left[\left\{\nu_{\alpha}, \bar{\rho}_{\alpha}, \bar{\omega}_{\alpha}\right\}\right]\right)
$$

where, for $\bar{\rho}_{\alpha}$ and $\bar{\omega}_{\alpha}$, the action is stationary, i. e.

$$
\begin{aligned}
\left.\frac{\delta \mathcal{H}\left[\left\{\nu_{\alpha}, \rho_{\alpha}, \omega_{\alpha}\right\}\right]}{\delta \rho_{\alpha}}\right|_{\left(\bar{\rho}_{\alpha}, \bar{\omega}_{\alpha}\right)} & \left.\frac{\delta \mathcal{H}\left[\left\{\nu_{\alpha}, \rho_{\alpha}, \omega_{\alpha}\right\}\right]}{\delta \omega_{\alpha}}\right|_{\left(\bar{\rho}_{\alpha}, \bar{\omega}_{\alpha}\right)} \\
& =0 .
\end{aligned}
$$

Replacing the CV action by its expression (21) in Eq. (43) leads to implicit equations for $\bar{\rho}_{\alpha}$ and $\bar{\omega}_{\alpha}$ :

$$
\begin{aligned}
\bar{\rho}_{\alpha}(1) & =\bar{\rho}_{\alpha}^{\mathrm{MF}}(1)=\rho_{\alpha}^{\mathrm{RS}}\left[\left\{\bar{\nu}_{\alpha}-\mathrm{i} \bar{\omega}_{\alpha}\right\}\right](1), \\
\mathrm{i} \bar{\omega}_{\alpha}(1) & =\Phi_{\alpha \beta}(1,2) \bar{\rho}_{\beta}(2),
\end{aligned}
$$

where $\rho_{\alpha}^{R S}\left[\left\{\bar{\nu}_{\alpha}-\mathrm{i} \bar{\omega}_{\alpha}\right\}\right](i)$ denotes the $\alpha$ th species number density of the RS fluid with the chemical potentials $\left\{\bar{\nu}_{\alpha}-\mathrm{i} \bar{\omega}_{\alpha}\right\}$. For a homogeneous system (44) can be rewritten in the form

$$
\begin{aligned}
\bar{\rho}_{\alpha} & =\bar{\rho}_{\alpha}^{\mathrm{MF}}=\rho_{\alpha}^{\mathrm{RS}}\left[\left\{\bar{\nu}_{\alpha}-\mathrm{i} \bar{\omega}_{\alpha}\right\}\right], \\
\mathrm{i} \bar{\omega}_{\alpha} & =\bar{\rho}_{\beta} \tilde{\Phi}_{\alpha \beta}(0),
\end{aligned}
$$

It follows from the stationary condition (43) that the MF density is given by

$$
\begin{aligned}
\rho_{\alpha}^{\mathrm{MF}}\left[\left\{\nu_{\alpha}\right\}\right](1) & =\frac{\delta \ln \Xi_{\mathrm{MF}}\left[\left\{\nu_{\alpha}\right\}\right]}{\delta \nu_{\alpha}(1)} \\
& =\rho_{\alpha}^{\mathrm{RS}}\left[\left\{\bar{\nu}_{\alpha}-\mathrm{i} \bar{\omega}_{\alpha}\right\}\right](1),
\end{aligned}
$$


and that the MF grand potential reads

$$
\begin{aligned}
\ln \Xi_{\mathrm{MF}}\left[\left\{\nu_{\alpha}\right\}\right] & =\ln \Xi_{\mathrm{RS}}\left[\left\{\bar{\nu}_{\alpha}-\mathrm{i} \bar{\omega}_{\alpha}\right\}\right] \\
& +\frac{\beta}{2}\left\langle\rho_{\alpha}^{\mathrm{MF}}\left|w_{\alpha \beta}\right| \rho_{\beta}^{\mathrm{MF}}\right\rangle .
\end{aligned}
$$

The MF Kohn-Scham free energy of a multicomponent system defined as the Legendre transform

$$
\beta \mathcal{A}_{\mathrm{MF}}\left[\left\{\rho_{\alpha}\right\}\right]=\sup _{\nu_{\alpha}}\left\{\left\langle\rho_{\alpha} \mid \nu_{\alpha}\right\rangle-\ln \Xi_{\mathrm{MF}}\left[\left\{\nu_{\alpha}\right\}\right]\right\},
$$

has the following form in the MF approximation

$$
\begin{aligned}
\beta \mathcal{A}_{\mathrm{MF}}\left[\left\{\rho_{\alpha}\right\}\right] & =\beta \mathcal{A}_{\mathrm{RS}}\left[\left\{\rho_{\alpha}\right\}\right] \\
& +\frac{\beta}{2}\left\langle\rho_{\alpha}\left|w_{\alpha \beta}\right| \rho_{\beta}\right\rangle-\frac{\beta}{2} \int_{\Omega} \mathrm{d} \mathbf{r} w_{\alpha \alpha}(0) \rho_{\alpha}(\mathbf{r}) .
\end{aligned}
$$

Using the formulas

$$
\begin{aligned}
& G_{\mathrm{MF}, \alpha_{1} \alpha_{2}}^{(2), T}\left[\left\{\nu_{\alpha}\right\}\right](1,2)=\frac{\delta^{2} \ln \Xi_{\mathrm{MF}}\left[\left\{\nu_{\alpha}\right\}\right]}{\delta \nu_{\alpha_{1}}(1) \delta \nu_{\alpha_{2}}(2)}, \\
& C_{\mathrm{MF}, \alpha_{1} \alpha_{2}}^{(2)}\left[\left\{\rho_{\alpha}\right\}\right](1,2)=-\frac{\delta^{2} \beta \mathcal{A}_{\mathrm{MF}}\left[\left\{\rho_{\alpha}\right\}\right]}{\delta \rho_{\alpha_{1}}(1) \delta \rho_{\alpha_{2}}(2)},
\end{aligned}
$$

where $(1,2, \ldots, n) \equiv\left(\mathbf{r}_{1}, \mathbf{r}_{2}, \ldots, \mathbf{r}_{n}\right)$, one can get the well-known expressions for the partial pair correlation and vertex (or direct correlation) functions in the MF approximation. $C_{\mathrm{MF}, \alpha_{1} \alpha_{2}}^{(2)}\left[\left\{\rho_{\alpha}\right\}\right](1,2)$ is obtained readily from expression (49)

$$
\begin{aligned}
C_{\mathrm{MF}, \alpha \beta}^{(2)}(1,2) & =-G_{\mathrm{MF}, \alpha \beta}^{(2), T-1}(1,2) \\
& =C_{\mathrm{RS}, \alpha \beta}^{(2)}(1,2)-w_{\alpha \beta}(1,2),
\end{aligned}
$$

where $C_{\mathrm{RS}, \alpha \beta}^{(2)}(1,2)$ means the exact two-point proper vertex of the RS fluid at the mean field density $\rho_{\alpha}^{\mathrm{MF}}$. The two-point vertex function $C_{\mathrm{MF}, \alpha \beta}^{(2)}$ is connected to the usual direct correlation function of the theory of liquids $c_{\mathrm{MF}, \alpha \beta}(1,2)$

$$
C_{\mathrm{MF}, \alpha \beta}^{(2)}(1,2)=c_{\mathrm{MF}, \alpha \beta}(1,2)-\frac{1}{\rho_{\alpha}(1)} \delta_{\alpha \beta} \delta(1,2) .
$$

In order to calculate $G_{\mathrm{MF}, \alpha \beta}^{(2), T}(1,2)$ we start with equation

$$
\begin{aligned}
G_{\mathrm{MF}, \alpha \beta}^{(2), T}(1,2) & =\frac{\partial \rho_{\alpha}^{\mathrm{MF}}\left[\left\{\bar{\nu}_{\alpha}-\mathrm{i} \bar{\omega}_{\alpha}\right\}\right](1)}{\partial \nu_{\beta}(2)} \\
& =\frac{\partial \rho_{\alpha}^{\mathrm{RS}}\left[\left\{\bar{\nu}_{\alpha}-\mathrm{i} \bar{\omega}_{\alpha}\right\}\right](1)}{\partial \nu_{\beta}(2)}
\end{aligned}
$$

$\rho_{\alpha}^{\mathrm{RS}}\left[\left\{\nu_{\alpha}\right\}\right]$ depends on $\nu_{\alpha}$ directly but also through the mean field $\bar{\omega}_{\alpha}$. Therefore, one has

$$
\begin{aligned}
G_{\mathrm{MF}, \alpha \beta}^{(2), T}(1,2) & =\left.\frac{\partial \rho_{\alpha}^{\mathrm{RS}}\left[\left\{\bar{\nu}_{\alpha}-\mathrm{i} \bar{\omega}_{\alpha}\right\}\right](1)}{\partial \nu_{\beta}(2)}\right|_{\bar{\omega}} \\
& +\left.\frac{\partial \rho_{\alpha}^{\mathrm{RS}}\left[\left\{\bar{\nu}_{\alpha}-\mathrm{i} \bar{\omega}_{\alpha}\right\}\right](1)}{\partial \bar{\omega}_{\gamma}(3)}\right|_{\nu_{\gamma}} \frac{\partial \bar{\omega}_{\gamma}(3)}{\partial \nu_{\beta}(2)}
\end{aligned}
$$

Taking into account (44) and (50) we obtain finally $G_{\mathrm{MF}, \alpha \beta}^{(2), T}(1,2)=G_{\mathrm{RS}, \alpha \beta}^{(2), T}(1,2)$

$$
-\beta G_{\mathrm{MF}, \alpha \gamma}^{(2), T}(1,3) w_{\gamma \delta}(3,4) G_{\mathrm{RS}, \delta \beta}^{T}(4,2) .
$$

(52) can be rewritten in a matricial form as [12]

$$
\begin{aligned}
\underline{G}_{\mathrm{MF}}^{(2), T}(1,2) & =\underline{G}_{\mathrm{RS}}^{(2), T}(1,2) \\
& -\underline{G}_{\mathrm{MF}}^{(2), T}(1,3) \underline{w}(3,4) \underline{G}_{\mathrm{RS}}^{(2), T}(4,2),
\end{aligned}
$$

where $\underline{G}_{\mathrm{MF}(\mathrm{RS})}^{(2), T}(i, j)$ denotes the matrix of elements $G_{\mathrm{MF}(\mathrm{RS}), \alpha \beta}^{(2), T}(i, j)$ and $\underline{w}(i, j)$ that of elements $\beta w_{\alpha \beta}(i, j)$. The formal solution of Eq. (53) is then

$$
\underline{G}_{\mathrm{MF}}^{(2), T}(1,2)=\left(\underline{1}+\underline{w} \star \underline{G}_{\mathrm{RS}}^{(2), T}\right)^{-1} \star \underline{G}_{\mathrm{RS}}^{(2), T}(1,2),
$$

where $\underline{1}=\delta_{\alpha \beta} \delta(1,2)$ is the unit operator and the " $\star$ " denotes a convolution in space.

\section{B. Beyond the MF approximation}

In order to take into account fluctuations we present $\operatorname{CVs} \rho_{\alpha}$ and $\omega_{\alpha}$ in the form:

$$
\rho_{\alpha}(1)=\bar{\rho}_{\alpha}+\delta \rho_{\alpha}(1), \quad \omega_{\alpha}(1)=\bar{\omega}_{\alpha}+\delta \omega_{\alpha}(1),
$$

where the quantities with a bar are given by (44).

The function $\ln \Xi_{\mathrm{RS}}\left[\left\{\bar{\nu}_{\alpha} ;-\mathrm{i} \omega_{\alpha}\right\}\right]$ in (14) can be presented in the form of the cumulant expansion

$$
\ln \Xi_{\mathrm{RS}}\left[\left\{\bar{\nu}_{\alpha}-\mathrm{i} \omega_{\alpha}\right\}\right]=\sum_{n \geq 1} \frac{(-\mathrm{i})^{n}}{n !} \sum_{\alpha_{1}, \ldots, \alpha_{n}} \int \mathrm{d} 1 \ldots \int \mathrm{d} n \mathfrak{M}_{\alpha_{1} \ldots \alpha_{n}}(1, \ldots, n) \delta \omega_{\alpha_{1}}(1) \ldots \delta \omega_{\alpha_{n}}(n)
$$

where $\mathfrak{M}_{\alpha_{1} \ldots \alpha_{n}}(1, \ldots, n)$ is the $n$th cumulant defined by

$$
\mathfrak{M}_{\alpha_{1} \ldots \alpha_{n}}(1, \ldots, n)=\left.\frac{1}{(-i)^{n}} \frac{\partial^{n} \ln \Xi_{\mathrm{RS}}\left[\left\{\bar{\nu}_{\alpha}-\mathrm{i} \omega_{\alpha}\right\}\right]}{\partial \delta \omega_{\alpha_{1}}(1) \ldots \partial \delta \omega_{\alpha_{n}}(n)}\right|_{\delta \omega_{\alpha_{i}}=0}
$$


As is seen from (56) and (25), the $n$th cumulant is equal to the $n$-particle partial truncated (connected) correlation function at $\omega_{\alpha}=\bar{\omega}_{\alpha}$. The expressions for the several cumulants given in the Cartesian coordinate phase space are as follows

$$
\begin{gathered}
\mathfrak{M}_{\alpha_{1}}(1)=\rho_{\alpha_{1}}(1) \\
\left.\mathfrak{M}_{\alpha_{1} \alpha_{2}}(1,2)=\rho_{\alpha_{1}}(1) \rho_{\alpha_{2}}(2) h_{\alpha_{1} \alpha_{2}}(1,2)+\rho_{\alpha_{1}}(1) \delta_{\alpha_{1} \alpha_{2}} \delta(1,2)\right] \\
\mathfrak{M}_{\alpha_{1} \alpha_{2} \alpha_{3}}(1,2,3)=\rho_{\alpha_{1}}(1) \rho_{\alpha_{2}}(2) \rho_{\alpha_{3}}(3) h_{\alpha_{1} \alpha_{2} \alpha_{3}}(1,2,3)+\rho_{\alpha_{1}}(1) \rho_{\alpha_{2}}(2) h_{\alpha_{1} \alpha_{2}}(1,2) \delta_{\alpha_{1} \alpha_{3}} \delta(1,3) \\
+\rho_{\alpha_{1}}(1) \rho_{\alpha_{3}}(3) h_{\alpha_{1} \alpha_{3}}(1,3) \delta_{\alpha_{1} \alpha_{2}} \delta(1,2)+\rho_{\alpha_{2}}(2) \rho_{\alpha_{3}}(3) h_{\alpha_{2} \alpha_{3}}(2,3) \delta_{\alpha_{1} \alpha_{2}} \delta(1,2) \\
+\rho_{\alpha_{1}}(1) \delta_{\alpha_{1} \alpha_{2}} \delta_{\alpha_{1} \alpha_{3}} \delta(1,2) \delta(1,3)
\end{gathered}
$$

$$
\begin{aligned}
\mathfrak{M}_{\alpha_{1} \alpha_{2} \alpha_{3} \alpha_{4}}(1,2,3,4) & =\rho_{\alpha_{1}}(1) \rho_{\alpha_{2}}(2) \rho_{\alpha_{3}}(3) \rho_{\alpha_{4}}(4) h_{\alpha_{1} \alpha_{2} \alpha_{3} \alpha_{4}}(1,2,3,4)+\sum_{i, j, k, l} \rho_{\alpha_{i}}(i) \rho_{\alpha_{j}}(j) \rho_{\alpha_{k}}(k) h_{\alpha_{i} \alpha_{j} \alpha_{k}}(i, j, k) \\
& \times \delta_{\alpha_{i} \alpha_{l}} \delta(i, l)+\sum_{i, j, k, l} \rho_{\alpha_{i}}(i) \rho_{\alpha_{k}}(k) h_{\alpha_{i} \alpha_{k}}(i, k) \delta_{\alpha_{i} \alpha_{j}} \delta_{\alpha_{k} \alpha_{l}} \delta(i, j) \delta(k, l)+\sum_{i, j, k, l} \rho_{\alpha_{i}}(i) \rho_{\alpha_{l}}(l) \\
& \times h_{\alpha_{i} \alpha_{l}}(i, l) \delta_{\alpha_{i} \alpha_{j}} \delta_{\alpha_{i} \alpha_{k}} \delta(i, j) \delta(i, k)+\rho_{\alpha_{1}}(1) \delta_{\alpha_{1} \alpha_{2}} \delta_{\alpha_{1} \alpha_{3}} \delta_{\alpha_{1} \alpha_{4}} \delta(1,2) \delta(1,3) \delta(1,4) .
\end{aligned}
$$

In the above formulas $\rho_{\alpha_{i}}(i)$ is the local density of the $\alpha_{i}$ th species in the RS and $h_{\alpha_{1} \ldots \alpha_{n}}(1, \ldots, n)$ is the $n$-particle partial correlation function of an $m$-component RS, defined in the GC ensemble (see Eqs. (28)-(29)):

$$
\begin{aligned}
h_{\alpha_{1} \alpha_{2}}(1,2) & =g_{\alpha_{1} \alpha_{2}}(1,2)-g_{\alpha_{1}}(1) g_{\alpha_{2}}(2) \\
h_{\alpha_{1} \alpha_{2} \alpha_{3}}(1,2,3) & =g_{\alpha_{1} \alpha_{2} \alpha_{3}}(1,2,3)-g_{\alpha_{1} \alpha_{2}}(1,2) g_{\alpha_{3}}(3) \\
& -g_{\alpha_{1} \alpha_{3}}(1,3) g_{\alpha_{2}}(2)-g_{\alpha_{2} \alpha_{3}}(2,3) g_{\alpha_{1}}(1)+2 g_{\alpha_{1}}(1) g_{\alpha_{2}}(2) g_{\alpha_{3}}(3) \\
h_{\alpha_{1} \alpha_{2} \alpha_{3} \alpha_{4}}(1,2,3,4) & =g_{\alpha_{1} \alpha_{2} \alpha_{3} \alpha_{4}}(1,2,3,4)-\ldots
\end{aligned}
$$

In the case of a homogeneous system a Fourier image of the $n$th cumulant can be presented in the form

$$
\mathfrak{M}_{\alpha_{1} \ldots \alpha_{n}}\left(\mathbf{k}_{1}, \ldots, \mathbf{k}_{n}\right)=\left(\left\langle N_{\alpha_{1}}\right\rangle, \ldots\left\langle N_{\alpha_{n}}\right\rangle\right)^{1 / n} S_{\alpha_{1} \ldots \alpha_{n}}\left(k_{1}, \ldots, k_{n}\right) \delta_{\mathbf{k}_{1}+\ldots+\mathbf{k}_{n}},
$$

where $S_{\alpha_{1} \ldots \alpha_{n}}\left(\mathbf{k}_{1}, \ldots, \mathbf{k}_{n}\right)$ is the $n$-particle partial structure factor of the RS.

Substituting (55) in (13) one can obtain

$$
\begin{aligned}
\Xi\left[\left\{\nu_{\alpha}\right\}\right] & =\Xi_{\mathrm{RS}}\left[\left\{\bar{\nu}_{\alpha}-\mathrm{i} \bar{\omega}_{\alpha}\right\}\right] \int \mathcal{D} \delta \rho \mathcal{D} \delta \omega \\
& \times \exp \left\{-\frac{\beta}{2}\left\langle\delta \rho_{\alpha}\left|w_{\alpha \beta}\right| \delta \rho_{\beta}\right\rangle+\mathrm{i}\left\langle\delta \omega_{\alpha} \mid \delta \rho_{\alpha}\right\rangle+\sum_{n \geq 2} \frac{(-\mathrm{i})^{n}}{n !} \sum_{\alpha_{1}, \ldots, \alpha_{n}} \int \mathrm{d} 1 \ldots \int \mathrm{d} n\right. \\
& \left.\times \mathfrak{M}_{\alpha_{1} \ldots \alpha_{n}}(1, \ldots, n) \delta \omega_{\alpha_{1}}(1) \ldots \delta \omega_{\alpha_{n}}(n)\right\} .
\end{aligned}
$$

Integrating in (63) over $\delta \omega_{\alpha_{i}}(i)$ we have in the homogeneous case

$$
\Xi\left[\left\{\nu_{\alpha}\right\}\right]=\Xi_{\mathrm{MF}} \Xi^{\prime} \int(\mathrm{d} \delta \rho) \exp \left\{-\frac{1}{2 !} \sum_{\alpha, \beta} \sum_{\mathbf{k}} \mathrm{L}_{\alpha \beta}(\mathrm{k}) \delta \rho_{\mathbf{k}, \alpha} \delta \rho_{-\mathbf{k}, \beta}+\sum_{\mathrm{n} \geq 3} \mathcal{H}_{\mathrm{n}}\left(\delta \rho_{\alpha}\right)\right\} .
$$


c. Gaussian approximation. In the Gaussian approximation, which corresponds to taking into account in (64) only the terms with $n \leq 2\left(\mathcal{H}_{n} \equiv 0\right)$, we have $L_{\alpha \beta}(k)=C_{\alpha \beta}(k)$, where $C_{\alpha \beta}(k)$ are the Fourier transforms of the partial direct correlation functions. After integrating in (64) we arrive at the GPF of an $m$ component system in the random phase approximation (RPA).

Using the Gaussian averages one can develop a loop expansion of $\Xi\left[\left\{\nu_{\alpha}\right\}\right]$ in the CV representation as it was done recently for a one-component fluid [13].

\section{CONCLUSION}

Using the CV method we have reconsidered the basic relations of statistical field theory of a multicomponent non-homogeneous fluid that follow from this approach. Contrary to the KSSHE theory [12] the corresponding CV action depends on two sets scalar fields: the field $\rho_{\alpha}$ connected to the number density of the $\alpha$ th species particles and the field $\omega_{\alpha}$ conjugated to $\rho_{\alpha}$. We derive the explicit expressions for the CV field correlations and obtain their relation to the density correlation functions of a multicomponent system.

Contrary to the theories based on the StratonovichHubbard transformation [2,3], the CV representation has some important advantages which could be very useful for more complicated models of fluids. In particular, it is valid for an arbitrary pair potential (including the pair interaction $w_{\alpha \beta}(1,2)$ which does not possess an inverse) and is easily generalized for the case of $n$-body interparticle interactions with $n>2$.
[1] I. A. Vakarchuk, I. F. Margolych, Teor. Mat. Fiz. 72, 462 (1987).

[2] R. L. Stratonovich, Sov. Phys. Solid State 2, 1824 (1958).

[3] J. Hubbard, Phys. Rev. Lett. 2, 77 (1959).

[4] D. N. Zubarev, Dokl. Acad. Nauk SSSR 95, 757 (1954).

[5] I. R. Yukhnovsky, Zh. Eksp. Teor. Fiz. 34, 379 (1958).

[6] D. Bohm, D. Pines, Phys. Rev. 82, 625 (1951)

[7] I. R. Yukhnovskii, Phase Transitions of the Second Order: Collective Variables Method (Singapore, World Scientific, 1987).

[8] I. R. Yukhnovskii, Proc. Steklov Inst. Math. 2, 223 (1992).

[9] O. V. Patsagan, I. R. Yukhnovskii, Teor. Mat. Fiz. 83, 72 (1990).

[10] I. R. Yukhnovskii, O. V. Patsahan, J. Stat. Phys. 81, 647 (1995).

[11] J.-M. Caillol, Mol. Phys. 101, 1617 (2003).

[12] J.-M. Caillol, J. Stat. Phys. 115, 1461 (2004).

[13] J.-M. Caillol, O. Patsahan, I. Mryglod, Physica A 368, 326 (2006)
[14] O. Patsahan, I. Mryglod, Condens. Matter Phys. 9, 659 (2006).

[15] I. R. Yukhnovskii, M. F. Holovko, Statistical Theory of Classical Equilibrium Systems (Naukova Dumka, Kiev, 1980).

[16] J. P. Hansen, I. R. McDonald, Theory of simple liquids (Academic Press, 1986).

[17] J. W. Negele, H. Orland, Quantum Many-Particle Systems (Frontiers in Physics, Addison-Wesley, 1988).

[18] F. J. Wegner, in Phase Transitions and Critical Phenomena, 6, edited by C. Domb, M. S. Green (Academic Press, New York, 1976), p. 7.

[19] J. Zinn-Justin, Quantum Field Theory and Critical Phenomena (Clarendon Press, Oxford, 1989).

[20] G. Stell, in Phase Transitionss and Critical Phenomena, $\mathbf{5 b}$, edited by C. Domb, M. S. Green (Academic Press, New York, 1973), p. 205.

[21] G. Stell, in Equilibrium theory of Classical Fluids, edited by H. L. Frisch, J. L. Lebowitz (North-Holland, Amsterdam, 1982), p. 171

\title{
СТАТИСТИКО-ПОЛЬОВА ТЕОРІЯ ДЛЯ БАГАТОКОМПОНЕНТНОГО ПЛИНУ: МЕТОД КОЛЕКТИВНИХ ЗМІННИХ
}

\author{
О. Пацаган ${ }^{1}$, І. Мриглод ${ }^{1}$, Ж.-М. Кайоль ${ }^{2}$ \\ ${ }^{1}$ Інститут фізики конденсованих систем НАН Украӥни \\ вул. Свенціцького, 1, Лъвів, 79011, Украӥна \\ 2 Лабораторія теоретичної фізики, \\ Університет Парі-Сюд, 91405, Орсей, Франція
}

\footnotetext{
Використовуючи метод колективних змінних (К3), розглянуто основні співвідношення статистикопольової теорії багатокомпонентної неоднорідної системи. Показано, що дія, отримана в представленні КЗ, залежить від двох наборів скалярних полів, а саме, полів $\rho_{\alpha}$, які описують флюктуації локальної густини частинок сорту $\alpha$ і полів $\omega_{\alpha}$, спряжених до $\rho_{\alpha}$. Знайдено явні вирази для кореляційних функцій полів K3, а також їх зв'язок із кореляційними функціями густини. Сформульовано теорію збурень і детально розглянуто наближення середнього поля.
} 